\title{
The Yagita Invariant of Symplectic Groups of Large Rank
}

\author{
Cornelia M. Busch and Ian J. Leary®
}

\begin{abstract}
Fix a prime $p$, and let $\mathcal{O}$ denote a subring of $\mathbb{C}$ that is either integrally closed or contains a primitive $p$ th root of 1 . We determine the Yagita invariant at the prime $p$ for the symplectic group $\operatorname{Sp}(2 n, \mathcal{O})$ for all $n \geq p-1$.
\end{abstract}

Mathematics Subject Classification. 20J05 (primary), 57T10 (secondary).

\section{Introduction}

The Yagita invariant $p^{\circ}(G)$ of a discrete group $G$ is an invariant that generalizes the period of the $p$-local Tate-Farrell cohomology of $G$, in the following sense: it is a numerical invariant defined for any $G$ that is equal to the period when the $p$-local cohomology of $G$ is periodic. Yagita considered finite groups [6], and Thomas extended the definition to groups of finite vcd [5]. In [3] the definition was extended to arbitrary groups and $p^{\circ}(G)$ was computed for $G=\operatorname{GL}(n, \mathcal{O})$ for $\mathcal{O}$ any integrally closed subring of $\mathbb{C}$ and for sufficiently large $n$ (depending on $\mathcal{O}$ ).

In [2], one of us computed the Yagita invariant for $\operatorname{Sp}(2(p+1), \mathbb{Z})$. Computations from [3] were used to provide an upper bound, and computations with finite subgroups and with mapping class groups were used to provide a lower bound [4]. The action of the mapping class group of a surface upon the first homology of the surface gives a natural symplectic representation of the mapping class group of a genus $p+1$ surface inside $\operatorname{Sp}(2(p+1), \mathbb{Z})$. In the current paper, we compute $p^{\circ}(\operatorname{Sp}(2 n, \mathcal{O}))$ for each $n \geq p-1$ for each $\mathcal{O}$ for which $p^{\circ}(\mathrm{GL}(n, \mathcal{O}))$ was computed in [3]. By using a greater range of finite subgroups, we avoid having to consider mapping class groups.

Cornelia M. Busch acknowledges support from ETH Zürich, which facilitated this work. Ian J. Leary would like to thank the Isaac Newton Institute for Mathematical Sciences for support and hospitality during the programme Non-positive Curvature, Group Actions and Cohomology, when work on this paper was undertaken. This work was supported by EPSRC Grant no. EP/K032208/1 and by a grant from the Leverhulme Trust RF-2016-321. 
Throughout the paper, we fix a prime $p$. Before stating our main result, we recall the definitions of the symplectic group $\operatorname{Sp}(2 n, R)$ over a ring $R$, and of the Yagita invariant $p^{\circ}(G)$, which depends on the prime $p$ as well as on the group $G$. The group $\operatorname{Sp}(2 n, R)$ is the collection of invertible $2 n \times 2 n$ matrices $M$ over $R$ such that

$$
M^{\mathrm{T}} J M=J, \text { where } J:=\left(\begin{array}{ll}
0 & I_{n} \\
-I_{n} & 0
\end{array}\right) .
$$

Here, $M^{\mathrm{T}}$ denotes the transpose of the matrix $M$, and as usual $I_{n}$ denotes the $n \times n$ identity matrix. Equivalently, $M \in \mathrm{Sp}(2 n, R)$ if $M$ defines an isometry of the antisymmetric bilinear form on $R^{2 n}$ defined by $\langle x, y\rangle:=x^{\mathrm{T}} J y$. If $C$ is cyclic of order $p$, then the group cohomology $\operatorname{ring} H^{*}(C ; \mathbb{Z})$ has the form

$$
H^{*}(C ; \mathbb{Z}) \cong \mathbb{Z}[x] /(p x), \quad x \in H^{2}(C ; \mathbb{Z}) .
$$

If $C$ is a cyclic subgroup of $G$ of order $p$, define $n(C)$ a positive integer or infinity to be the supremum of the integers $n$ such that the image of $H^{*}(G ; \mathbb{Z}) \rightarrow H^{*}(C ; \mathbb{Z})$ is contained in the subring $\mathbb{Z}\left[x^{n}\right]$. Now, define

$$
p^{\circ}(G):=\operatorname{lcm}\{2 n(C): C \leq G,|C|=p\} .
$$

It is easy to see that if $H \leq G$, then $p^{\circ}(H)$ divides $p^{\circ}(G)$ [3, Prop. 1].

\section{Results}

In the following theorem statement and throughout the paper, we let $\zeta_{p}$ be a primitive $p$ th root of 1 in $\mathbb{C}$ and we let $\mathcal{O}$ denote a subring of $\mathbb{C}$ with $F \subseteq \mathbb{C}$ as its field of fractions. We assume that either $\zeta_{p} \in \mathcal{O}$ or that $\mathcal{O}$ is integrally closed in $\mathbb{C}$. We define $l:=\left|F\left[\zeta_{p}\right]: F\right|$, the degree of $F\left[\zeta_{p}\right]$ as an extension of $F$. For $t \in \mathbb{R}$ with $t \geq 1$, we define $\psi(t)$ to be the largest integer power of $p$ less than or equal to $t$.

Theorem 1. With notation as above, for each $n \geq p-1$, the Yagita invariant $p^{\circ}(\operatorname{Sp}(2 n, \mathcal{O}))$ is equal to $2(p-1) \psi(2 n / l)$ for $l$ even and equal to $2(p-1) \psi(n / l)$ for $l$ odd.

By the main result of [3], the above is equivalent to the statement that $p^{\circ}(\operatorname{Sp}(2 n, \mathcal{O}))=p^{\circ}(\operatorname{GL}(2 n, \mathcal{O}))$ when $l$ is even and $p^{\circ}(\operatorname{Sp}(2 n, \mathcal{O}))=$ $p^{\circ}(\mathrm{GL}(n, \mathcal{O}))$ when $l$ is odd. By definition $\operatorname{Sp}(2 n, \mathcal{O})$ is a subgroup of $\operatorname{GL}(2 n, \mathcal{O})$ and there is an inclusion $\operatorname{GL}(n, \mathcal{O}) \rightarrow \operatorname{Sp}(2 n, \mathcal{O})$ defined by

$$
A \mapsto\left(\begin{array}{ll}
A & 0 \\
0 & \left(A^{\mathrm{T}}\right)^{-1}
\end{array}\right),
$$

and so for any $n, p^{\circ}(\mathrm{GL}(n, \mathcal{O}))$ divides $p^{\circ}(\operatorname{Sp}(2 n, \mathcal{O}))$, which in turn divides $p^{\circ}(\mathrm{GL}(2 n, \mathcal{O}))$.

Before we start, we recall two standard facts concerning symplectic matrices that will be used in the proof of Corollary 3: if $M$ is in the symplectic group, then $\operatorname{det}(M)=1$ and $M$ is conjugate to the inverse of its transpose $\left(M^{-1}\right)^{\mathrm{T}}=\left(M^{\mathrm{T}}\right)^{-1}$. We shall use the notation $\mathbb{F}_{p}^{\times}$to denote the multiplicative group of units in the field $\mathbb{F}_{p}$. 
Proposition 2. Let $f(X)$ be a polynomial over the field $\mathbb{F}_{p}$ and suppose that 0 is not a root of $f$, but that $f$ factors as a product of linear polynomials over $\mathbb{F}_{p}$. If there is a polynomial $g$ and an integer $n$ so that $f(X)=g\left(X^{n}\right)$, then $n$ has the form $n=m p^{q}$ for some $m$ dividing $p-1$ and some integer $q \geq 0$. If $p$ is odd and for each $i \in \mathbb{F}_{p}^{\times}$, the multiplicity of $i$ as a root of $f$ is equal to that of $-i$, then $m$ is even.

Proof. The only part of this that is not contained in [3, Prop. 6] is the final statement. Since $(1-i X)(1+i X)=1-i^{2} X^{2}$ is a polynomial in $X^{2}$, the final statement follows. For the benefit of the reader, we sketch the rest of the proof. If $n=m p^{q}$ where $p$ does not divide $m$, then $g\left(X^{n}\right)=g\left(X^{m}\right)^{p^{q}}$, so we may assume that $q=0$. If $g(Y)=0$ has roots $y_{i}$, then the roots of $g\left(X^{m}\right)=0$ are the roots of $y_{i}-X^{m}=0$. Since $p$ does not divide $m$, these polynomials have no repeated roots; since their roots are assumed to lie in $\mathbb{F}_{p}$ it is now easy to show that $m$ divides $p-1$.

Corollary 3. With notation as in Theorem 1 , let $G$ be a subgroup of $\operatorname{Sp}(2 n, F)$. Then the Yagita invariant $p^{\circ}(G)$ divides the number given for $p^{\circ}(\operatorname{Sp}(2 n, \mathcal{O}))$ in the statement of Theorem 1.

Proof. As in [3, Cor. 7], for each $C \leq G$ of order $p$, we use the total Chern class to give an upper bound for the number $n(C)$ occurring in the definition of $p^{\circ}(G)$. If $C$ is cyclic of order $p$, then $C$ has $p$ distinct irreducible complex representations, each one dimensional. If we write $H^{*}(C ; \mathbb{Z})=\mathbb{Z}[x] /(p x)$, then the total Chern classes of these representations are $1+i x$ for each $i \in \mathbb{F}_{p}$, where $i=0$ corresponds to the trivial representation. The total Chern class of a direct sum of representations is the product of the total Chern classes, and so when viewed as a polynomial in $\mathbb{F}_{p}[x]=H^{*}(C ; \mathbb{Z}) \otimes \mathbb{F}_{p}$, the total Chern class of any faithful representation $\rho: C \rightarrow \mathrm{GL}(2 n, \mathbb{C})$ is a non-constant polynomial of degree at most $2 n$ all of whose roots lie in $\mathbb{F}_{p}^{\times}$. Now, let $F$ be a subfield of $\mathbb{C}$ with $l=\left|F\left[\zeta_{p}\right]: F\right|$ as in the statement. The group $C$ has $(p-1) / l$ non-trivial irreducible representations over $F$, each of dimension $l$, and the total Chern classes of these representations have the form $1-i x^{l}$, where $i$ ranges over the $(p-1) / l$ distinct $l$ th roots of unity in $\mathbb{F}_{p}$. In particular, the total Chern class of any representation $\rho: C \rightarrow \mathrm{GL}(2 n, F) \leq \mathrm{GL}(2 n, \mathbb{C})$ is a polynomial in $x^{l}$ whose $x$-degree is at most $2 n$. If $\rho$ has image contained in $\operatorname{Sp}(2 n, \mathbb{C})$, then it factors as $\rho=\iota \circ \widetilde{\rho}$ with $\widetilde{\rho}: C \rightarrow \operatorname{Sp}(2 n, \mathbb{C})$ and $\iota$ is the inclusion of $\operatorname{Sp}(2 n, \mathbb{C})$ in $\mathrm{GL}(2 n, \mathbb{C})$. In this case, the matrix representing a generator for $C$ is conjugate to the transpose of its own inverse; in particular, it follows that the multiplicities of the irreducible complex representations of $C$ with total Chern classes $1+i x$ and $1-i x$ must be equal for each $i$. Hence in this case, if $p$ is odd, the total Chern class of the representation $\rho=\iota \circ \widetilde{\rho}$ is a polynomial in $x^{2}$. If $p=2$ (which implies that $l=1$ ), then the total Chern class of any representation $\rho: C \rightarrow \operatorname{GL}(2 n, \mathbb{C})$ has the form $(1+x)^{i}$, where $i$ is equal to the number of non-trivial irreducible summands. Since $\operatorname{Sp}(2 n, \mathbb{C}) \leq \mathrm{SL}(2 n, \mathbb{C})$, it follows that for symplectic representations $i$ must be even, and so for $p=2$ the total Chern class is a polynomial in $x^{2}$.

In summary, let $\widetilde{\rho}$ be a faithful representation of $C$ in $\operatorname{Sp}(2 n, F)$. In the case when $l$ is odd, then the total Chern class of $\tilde{\rho}$ is a non-constant 
polynomial $f(y) \stackrel{\sim}{=} f(x)$ in $y=x^{2 l}$ such that $f(x)$ has degree at most $2 n$, $\tilde{f}(y)$ has degree at most $n / l$, and all roots of $f, \tilde{f}$ lie in $\mathbb{F}_{p}^{\times}$. In the case when $l$ is even, the total Chern class of $\rho$ is a non-constant polynomial $\tilde{f}(y)=f(x)$ in $y=x^{l}$ such that $f(x)$ has degree at most $2 n, \tilde{f}(y)$ has degree at most $2 n / l$, and all roots of both lie in $\mathbb{F}_{p}^{\times}$. By Proposition 2, it follows that each $n(C)$ is a factor of the number given for $p^{\circ}(\operatorname{Sp}(2 n, \mathcal{O}))$, and hence the claim.

Lemma 4. Let $H \leq G$ with $|G: H|=m$, and let $\rho$ be a symplectic representation of $H$ on $V=\mathcal{O}^{2 n}$. The induced representation $\operatorname{Ind}_{H}^{G}(\rho)$ is a symplectic representation of $G$ on $W:=\mathcal{O} G \otimes_{\mathcal{O} H} V \cong \mathcal{O}^{2 m n}$.

Proof. Let $e_{1}, \ldots, e_{n}, f_{1}, \ldots, f_{n}$ be the standard basis for $V=\mathcal{O}^{2 n}$, so that the bilinear form $\langle v, w\rangle:=v^{\mathrm{T}} J w$ on $V$ is given by

$$
\left\langle e_{i}, e_{j}\right\rangle=0=\left\langle f_{i}, f_{j}\right\rangle,\left\langle e_{i}, f_{j}\right\rangle=-\left\langle f_{i}, e_{j}\right\rangle=\delta_{i j} .
$$

The representation $\rho$ is symplectic if and only if each $\rho(h)$ preserves this bilinear form.

Let $t_{1}, \ldots, t_{m}$ be a left transversal to $H$ in $G$, so that $\mathcal{O} G=\oplus_{i=1}^{m} t_{i} \mathcal{O} H$ as right $\mathcal{O} H$-modules. Define a bilinear form $\langle,\rangle_{W}$ on $W$ by

$$
\left\langle\sum_{i=1}^{m} t_{i} \otimes v^{i}, \sum_{i=1}^{m} t_{i} \otimes w^{i}\right\rangle_{W}:=\sum_{i=1}^{m}\left\langle v^{i}, w^{i}\right\rangle .
$$

To see that this bilinear form is preserved by the $\mathcal{O} G$-action on $W$, fix $g \in G$ and define a permutation $\pi$ of $\{1, \ldots, m\}$ and elements $h_{1}, \ldots, h_{m} \in H$ by the equations $g t_{i}=t_{\pi(i)} h_{i}$. Now for each $i, j$ with $1 \leq i, j \leq m$,

$$
\begin{aligned}
\left\langle\operatorname{Ind}(\rho(g)) t_{i} \otimes v, \operatorname{Ind}(\rho(g)) t_{j} \otimes w\right\rangle_{W} & =\left\langle t_{\pi(i)} \otimes \rho\left(h_{i}\right) v, t_{\pi(j)} \otimes \rho\left(h_{j}\right) w\right\rangle_{W} \\
& =\delta_{\pi(i) \pi(j)}\left\langle\rho\left(h_{i}\right) v, \rho\left(h_{j}\right) w\right\rangle \\
& =\delta_{i j}\left\langle\rho\left(h_{i}\right) v, \rho\left(h_{i}\right) w\right\rangle \\
& =\delta_{i j}\langle v, w\rangle \\
& =\left\langle t_{i} \otimes v, t_{j} \otimes w\right\rangle_{W} .
\end{aligned}
$$

To see that $\langle,\rangle_{W}$ is symplectic, define basis elements $E_{1}, \ldots, E_{m n}, F_{1}, \ldots, F_{m n}$ for $W$ by the equations

$E_{n(i-1)+j}:=t_{i} \otimes e_{j}$, and $F_{n(i-1)+j}:=t_{i} \otimes f_{j}$, for $1 \leq i \leq m, 1 \leq j \leq n$.

It is easily checked that for $1 \leq i, j \leq m n$

$$
\left\langle E_{i}, E_{j}\right\rangle_{W}=0=\left\langle F_{i}, F_{j}\right\rangle_{W},\left\langle E_{i}, F_{j}\right\rangle_{W}=-\left\langle F_{i}, E_{j}\right\rangle_{W}=\delta_{i j},
$$

and so with respect to this basis for $W$, the bilinear form $\langle,\rangle_{W}$ is the standard symplectic form.

Proposition 5. With notation as in Theorem 1, the Yagita invariant $p^{\circ}(\operatorname{Sp}(2 n, \mathcal{O}))$ is divisible by the number given in the statement of Theorem 1 .

Proof. To give lower bounds for $p^{\circ}(\operatorname{Sp}(2 n, \mathcal{O}))$, we use finite subgroups. Firstly, consider the semidirect product $H=C_{p} \rtimes C_{p-1}$, where $C_{p-1}$ acts faithfully on $C_{p}$; equivalently, this is the group of affine transformations of the line over $\mathbb{F}_{p}$. It is well known that the image of $H^{*}(G ; \mathbb{Z})$ inside $H^{*}\left(C_{p} ; \mathbb{Z}\right) \cong \mathbb{Z}[x] /(p x)$ 
is the subring generated by $x^{p-1}$. It follows that $2(p-1)$ divides $p^{\circ}(G)$ for any $G$ containing $H$ as a subgroup. The group $H$ has a faithful permutation action on $p$ points, and hence a faithful representation in $\operatorname{GL}(p-1, \mathbb{Z})$, where $\mathbb{Z}^{p-1}$ is identified with the kernel of the $H$-equivariant map $\mathbb{Z}\{1, \ldots, p\} \rightarrow \mathbb{Z}$. Since $\mathrm{GL}(p-1, \mathbb{Z})$ embeds in $\operatorname{Sp}(2(p-1), \mathbb{Z})$, we deduce that $H$ embeds in $\operatorname{Sp}(2 n, \mathcal{O})$ for each $\mathcal{O}$ and for each $n \geq p-1$.

To give a lower bound for the $p$-part of $p^{\circ}(\operatorname{Sp}(2 n, \mathcal{O}))$, we use the extraspecial $p$-groups. For $p$ odd, let $E(p, 1)$ be the non-abelian $p$-group of order $p^{3}$ and exponent $p$, and let $E(2,1)$ be the dihedral group of order 8. (Equivalently in each case $E(p, 1)$ is the Sylow $p$-subgroup of $\mathrm{GL}\left(3, \mathbb{F}_{p}\right)$.) For $m \geq 2$, let $E(p, m)$ denote the central product of $m$ copies of $E(p, 1)$, so that $E(p, m)$ is one of the two extraspecial groups of order $p^{2 m+1}$. Yagita showed that $p^{\circ}(E(p, m))=2 p^{m}$ for each $m$ and $p$ [6]. The centre and commutator subgroup of $E(p, m)$ are equal and have order $p$, and the abelianization of $E(p, m)$ is isomorphic to $C_{p}^{2 m}$. The irreducible complex representations of $E(p, m)$ are well understood: there are $p^{2 m}$ distinct one-dimensional irreducibles, each of which restricts to the centre as the trivial representation, and there are $p-1$ faithful representations of dimension $p^{m}$, each of which restricts to the centre as the sum of $p^{m}$ copies of a single (non-trivial) irreducible representation of $C_{p}$. The group $G=E(p, m)$ contains a subgroup $H$ isomorphic to $C_{p}^{m+1}$, and each of its faithful $p^{m}$-dimensional representations can be obtained by inducing up a one-dimensional representation $H \rightarrow C_{p} \rightarrow \mathrm{GL}(1, \mathbb{C})$.

According to Bürgisser, $C_{p}$ embeds in $\operatorname{Sp}(2 l, \mathcal{O})$ (resp. in $\operatorname{Sp}(l, \mathcal{O})$ when $l$ is even) provided that $\mathcal{O}$ is integrally closed in $\mathbb{C}[1]$. Here as usual, $l:=$ $\left|F\left[\zeta_{p}\right], F\right|$ and $F$ is the field of fractions of $\mathcal{O}$. If instead $\zeta_{p} \in \mathcal{O}$, then $l=1$ and clearly $C_{p}$ embeds in $\operatorname{GL}(1, \mathcal{O})$ and hence also in $\operatorname{Sp}(2, \mathcal{O})=\operatorname{Sp}(2 l, \mathcal{O})$. Taking this embedding of $C_{p}$ and composing it with any homomorphism $H \rightarrow C_{p}$, we get a symplectic representation $\rho$ of $H$ on $\mathcal{O}^{2 l}$ for any $l$ (resp. on $\mathcal{O}^{l}$ for $l$ even). For a suitable homomorphism, we know that $\operatorname{Ind}_{H}^{G}(\rho)$ is a faithful representation of $G$ on $\mathcal{O}^{2 l p^{m}}$ (resp. on $\mathcal{O}^{l p^{m}}$ for $l$ even) and by Lemma 4 we see that $\operatorname{Ind}_{H}^{G}(\rho)$ is symplectic. Hence, we see that $E(m, p)$ embeds as a subgroup of $\operatorname{Sp}\left(2 l p^{m}, \mathcal{O}\right)$ for any $l$ and as a subgroup of $\operatorname{Sp}\left(l p^{m}, \mathcal{O}\right)$ in the case when $l$ is even. Since $p^{\circ}(E(m, p))=2 p^{m}$, this shows that $2 p^{m}$ divides $p^{\circ}\left(\operatorname{Sp}\left(2 l p^{m}, \mathcal{O}\right)\right)$ always and that $2 p^{m}$ divides $p^{\circ}\left(\operatorname{Sp}\left(l p^{m}, \mathcal{O}\right)\right)$ in the case when $l$ is even.

Corollary 3 and Proposition 5 together complete the proof of Theorem 1.

We finish by pointing out that we have not computed $p^{\circ}(\operatorname{Sp}(2 n, \mathcal{O}))$ for general $\mathcal{O}$ when $n<p-1$; to do this one would have to know which metacyclic groups $C_{p} \rtimes C_{k}$ with $k$ coprime to $p$ admit low-dimensional symplectic representations.

Open Access. This article is distributed under the terms of the Creative Commons Attribution 4.0 International License (http://creativecommons.org/licenses/by/4. $0 /$, which permits unrestricted use, distribution, and reproduction in any medium, 
provided you give appropriate credit to the original author(s) and the source, provide a link to the Creative Commons license, and indicate if changes were made.

Publisher's Note Springer Nature remains neutral with regard to jurisdictional claims in published maps and institutional affiliations.

\section{References}

[1] Bürgisser, B.: Elements of finite order in symplectic groups. Arch. Math. (Basel) 39(6), 501-509 (1982)

[2] Busch, C.M.: The Yagita invariant of some symplectic groups. Mediterr. J. Math. 10(1), 137-146 (2013)

[3] Glover, H.H., Leary, I.J., Thomas, C.B.: The Yagita Invariant of general linear groups. Algebraic topology: new trends in localization and periodicity (Sant Feliu de Guíxols, 1994). Progr. Math. 136, 185-192 (1996)

[4] Glover, H.H., Mislin, G., Xia, Y.: On the Yagita invariant of mapping class groups. Topology 33(3), 557-574 (1994)

[5] Thomas, C.B.: Free actions by $p$-groups on products of spheres and Yagita's invariant $p o(G)$. In: Transformation groups (Osaka, 1987), Lecture Notes in Math., vol. 1375, pp. 326-338. Springer, Berlin (1989)

[6] Yagita, N.: On the dimension of spheres whose product admits a free action by a non-abelian group. Q. J. Math. Oxford Ser. (2) 36(141), 117-127 (1985)

Cornelia M. Busch

Department of Mathematics

ETH Zürich

Rämistrasse 101

8092 Zurich

Switzerland

e-mail: cornelia.busch@math.ethz.ch

Ian J. Leary

School of Mathematical Sciences

University of Southampton

Southampton SO17 1BJ

UK

e-mail: i.j.leary@soton.ac.uk

Received: March 26, 2018.

Revised: April 26, 2019.

Accepted: October 9, 2019. 